\title{
Effect of oxygen on the threshold voltage of a-IGZO TFT
}

\author{
Eugene Chong**, Yoon Soo Chun*, Seung Han Kim* and Sang Yeol Lee ${ }^{\dagger}$
}

\begin{abstract}
Thin-film transistors (TFTs) are fabricated using an amorphous indium gallium zinc oxide (a-IGZO) channel layer by rf-magnetron sputtering. Oxygen partial pressure significantly changed the transfer characteristics of a-IGZO TFTs. Measurements performed on a-IGZO TFT show the change of threshold voltage in the transistor channel layer and electrical properties with varying $\mathrm{O}_{2}$ ratios. The device performance is significantly affected by adjusting the $\mathrm{O}_{2}$ ratio. This ratio is closely related with the modulation generation by reducing the localized trapping carriers and defect centers at the interface or in the channel layer.
\end{abstract}

Keywords: a-IGZO, Oxide TFT, $\mathrm{O}_{2}$ partial pressure, Threshold voltage

\section{Introduction}

Zn-based oxides are among the oldest materials because their constituents are easily accessible in copious amounts. In the semiconductor study for TFTs, various research groups have focused on amorphous oxide semiconductors based on the adoption of metal cation species [1-7]. The primary demands for such oxide-based semiconductors are: high optical band gap $(>3.5 \mathrm{eV})$, biocompatibility, nontoxicity, good tailored electrical conductivity, high optical transparency ( $>80 \%$ in VIS-IR), good ability for etching; lithography process compatibility, surface uniformity, and high thermal and chemical stability in various environments [8-10]. These demands are due to technological developments achieved in the past several years. Amorphous In-Ga-Zn-O (a-IGZO), one of the most attractive materials, has been investigated tremendously by many researchers for its practical applications. This is because a-IGZO has superb optical and electrical properties. In particular, its electrical resistivity is wideranging $\left(10^{10}-10^{-4} \Omega\right)$ because the ns-orbital of the metal cation is larger than the 2p-orbital of oxygen anion [11]. Thus, many studies have mainly focused on controlling the performance of the a-IGZO device, which is dependent on various susceptible parameters such as sputtering power and post-treatment [12-14]. The thickness of the channel layer can also be an important parameter to control device performance. Specifically, it can effectively enhance the switching behavior and threshold voltage $\left(V_{t h}\right)$ of TFTs through proper semiconductor resistance path adjustment $[2,15]$. The properties of a-IGZO TFTs are highly

$\dagger$ Corresponding Author: Nanoelectronics, University of Science and Technology, Daejeon, 305-333, Republic of Korea. (1sy@kist.re.kr)

* Electronic Materials Center, Korea Institute of Science and Technology, Seoul 136-791, Republic of Korea.

** Nanoelectronics, University of Science and Technology, Daejeon, 305-333, Republic of Korea.

Received: May 14, 2010; Accepted: March 29, 2011 dependent on their oxygen content, but in an interactive way; this is because oxygen vacancies provide the needed free carriers for electrical conduction [3]. In fact, oxygen vacancy can be easily generated in the oxides. Therefore, the electrical properties cannot be controlled easily. In other words, the carriers are generated easily from oxygen vacancies according to the following equation [16]:

$$
O_{0}^{x} \rightarrow 1 / 2 O_{2}(g)+V_{O}^{\prime \prime}=2 e^{\prime}
$$

However, the presentation of the second metal ion in the main matrix enhances the film's intensity to oxygen that can control electrical properties [7]. In the present work, the $\mathrm{O}_{2}$ concentration effect on a-IGZO is described for the control of the threshold voltage $\left(V_{t h}\right)$ parameter due to varying $\mathrm{O}_{2}$ partial pressure in a narrow range. This involves understanding how oxygen ratios influence the electronic properties and how the electronic behavior can be controlled.

\section{Experiments}

The a-IGZO films were placed on an $\mathrm{SiO}_{2}(100 \mathrm{~nm}) / \mathrm{Si}$ substrate through conventional rf-magnetron sputtering. The $O_{2}$ gas content varied from $1.4 \%-1.8 \%$ to control $V_{t h}$ of the a-IGZO films, because $O$ vacancies are known to be shallow donors located in the $\mathrm{ZnO}$-based system [17, 18]. The a-IGZO film $(50 \mathrm{~nm})$ was deposited thermally on oxidized silicon wafers as the channel layer for TFTs. The bottom gate and top electrode TFTs were fabricated on $\mathrm{SiO}_{2}$ (gate insulator, $100 \mathrm{~nm}$ ) $/ \mathrm{Si}$ substrates, and $\mathrm{Au}(80$ $\mathrm{nm}) / \mathrm{Ti}(10 \mathrm{~nm})$ layers were used as the source and drain electrodes. As-fabricated TFTs were annealed at $350{ }^{\circ} \mathrm{C}$ for $60 \mathrm{~min}$ in solely $\mathrm{Ar}$ ambience [19]. The amorphous structure was confirmed by X-ray diffraction, after which cross-sectional TEM and selected area electron diffraction 
observations were carried out. All electrical characterizations were performed using a semiconductor parameter analyzer in the dark at room temperature. The measured devices have an electrode width of $50 \mu \mathrm{m}$ and a channel length of $100 \mu \mathrm{m}$.

\section{Discussion}

Fig. 1 shows the XRD patterns of the IGZO film with different $O_{2}$ ratios. The films were found to be amorphous regardless of varied $\mathrm{O}_{2}$ ratios. In addition, the TEM and SEM images of the films revealed a very smooth surface [Figs. 2(a) and (b)]. This indicates that the IGZO film is amorphous. In the case of the polycrystalline $\mathrm{ZnO}$-based thin film, the crystallinity of the film was affected by oxygen contents. The grain shape and size also changed. The grain sizes decreased as the oxygen gas content increased [20]. The measured electrical properties are summarized in Table 1.

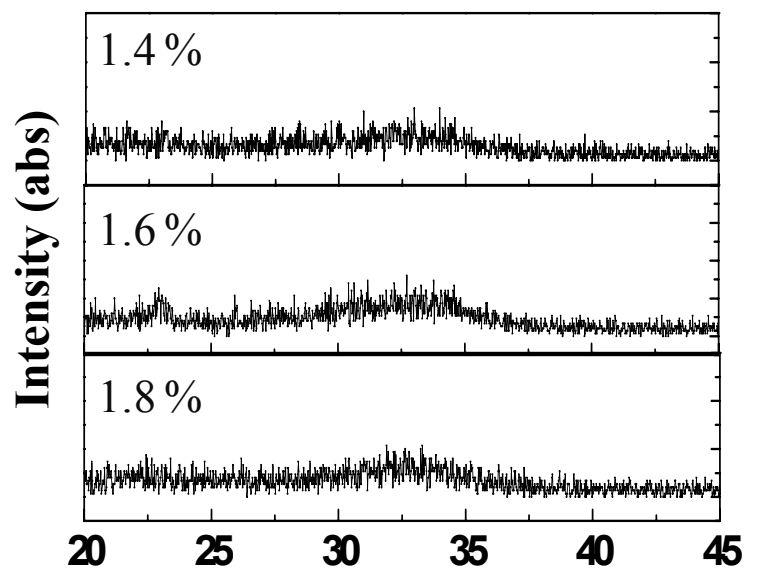

Fig. 1. X-ray diffraction peaks of IGZO film are dependent on $\mathrm{O}_{2}$ partial pressure at an $\mathrm{rf}$ power density of 5.09 $\mathrm{W} / \mathrm{cm}^{2}$

Table 1. Electrical properties of a-IGZO TFTs as a function of $\mathrm{O}_{2}$ partial pressure variation when the power density was set to 2.55 and $5.09 \mathrm{~W} / \mathrm{cm}^{2}$

\begin{tabular}{c|c|c|c|c}
\hline $\begin{array}{c}\text { Power density } \\
\left(\mathrm{W} / \mathrm{cm}^{2}\right)\end{array}$ & $\begin{array}{c}\mathrm{O}_{2} \\
\text { ratio } \\
(\%)\end{array}$ & $\begin{array}{c}\mathrm{V}_{\text {th }} \\
(\mathrm{V})\end{array}$ & $\begin{array}{c}\mathrm{I}_{\text {on }} \\
(\mathrm{A})\end{array}$ & $\begin{array}{c}\mathrm{I}_{\mathrm{On} / \text { off }} \\
\text { ratio }\end{array}$ \\
\hline \multirow{3}{*}{2.55} & 1.4 & 6.2 & $1.0 \times 10^{5}$ & $3.1 \times 10^{5}$ \\
\cline { 2 - 5 } & 1.6 & 7.3 & $1.3 \times 10^{5}$ & $7.5 \times 10^{5}$ \\
\cline { 2 - 5 } & 1.8 & 9.2 & $2.2 \times 10^{5}$ & $1.5 \times 10^{6}$ \\
\hline \multirow{3}{*}{5.09} & 1.4 & 2.4 & $2.5 \times 10^{6}$ & $1.0 \times 10^{6}$ \\
\cline { 2 - 5 } & 1.6 & 3.8 & $7.4 \times 10^{6}$ & $4.9 \times 10^{6}$ \\
\cline { 2 - 5 } & 1.8 & 9.1 & $3.0 \times 10^{6}$ & $1.8 \times 10^{6}$ \\
\hline
\end{tabular}

In the case of a-IGZO TFT at an rf power density of 2.55 $\mathrm{W} / \mathrm{cm}^{2}$, the $V_{t h}$ on current $I_{o n}$ and the on-to-off ratios were measured to about $6.2-9.2 \mathrm{~V},>10^{5} \mathrm{~A}$ and $\sim 1.5 \times 10^{6}$, respectively, depending on $\mathrm{O}_{2}$ partial pressure. For the aIGZO TFT at an rf power density of $5.09 \mathrm{~W} / \mathrm{cm}^{2}$, the $V_{t h}$,
$I_{o n}$, and on-to-off ratios were measured to about $2.4-9.1 \mathrm{~V}$, $>10^{6} \mathrm{~A}$ and $\sim 4.9 \times 10^{6}$, respectively, also depending on $O_{2}$ partial pressure. The representative transfer characteristic of a-IGZO TFTs with varying $\mathrm{O}_{2}$ partial pressure levels and drain-to-source voltages $\left(V_{D S}\right)=10 \mathrm{~V}$ are shown in Figs. 3 and 4. All of the TFTs are n-channel and operate in enhancement mode (i.e., $V_{t h}>0$ ). The $\mathrm{V}_{t h}$ was defined by linearly fitting the square root of $I_{D}$ vs. $V_{G}$ curve of the transistor in the saturation region. This can be expressed as follows:

$$
I_{D}=\left(\frac{C_{i} \mu_{s a t} W}{2 L}\right)\left(V_{G}-V_{t h}\right)^{2} \quad \text { for } \quad V_{D}>V_{G}-V_{t h}
$$

$W$ is the channel width, $L$ is the channel length, and $C_{i}$ is the capacitance per unit area of the gate-insulator. Interestingly, the $V_{\text {th }}$ value conspicuously shifted in the positive direction, from 2.4 to $9.1 \mathrm{~V}$ as the $\mathrm{O}_{2}$ ratio increased from $1.4 \%$ to $1.8 \%$ at an rf power density of 5.09 $\mathrm{W} / \mathrm{cm}^{2}$. The dependence of the transfer characteristics as a function of $\mathrm{O}_{2}$ partial pressure at an $\mathrm{rf}$ power density of $2.55 \mathrm{~W} / \mathrm{cm}^{2}$ is shown in Fig. 2. The $V_{t h}$ behavior was observed to be the same for a-IGZO TFTs at both rf power densities of $2.55 \mathrm{~W} / \mathrm{cm}^{2}$ and $5.09 \mathrm{~W} / \mathrm{cm}^{2}$. The possible mechanism of $\Delta V_{t h}$ is shown schematically in Fig. 5. The carrier concentration changed. This is due to fact that the drain current change was calculated from the relation $n=I D L / q V D \mu_{F E} W d$, where $n$ is the carrier concentration, $\mathrm{I}_{\mathrm{D}}$

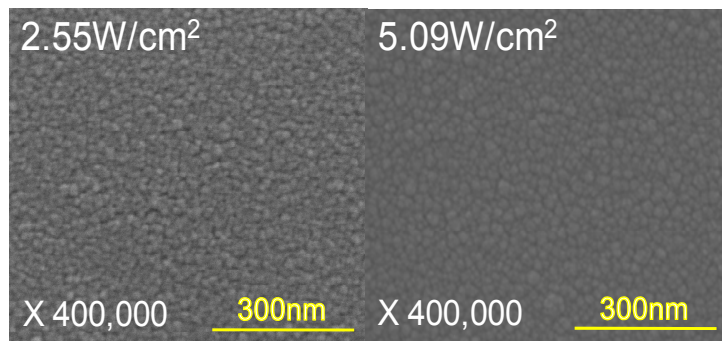

(a)

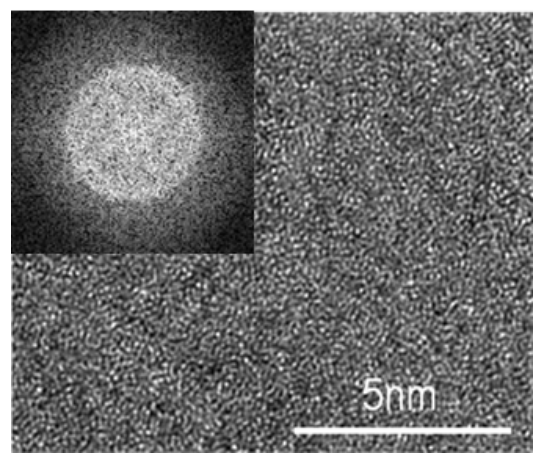

(b)

Fig. 2. (a) SEM image and (b) TEM cross-section image of the a-IGZO films. 


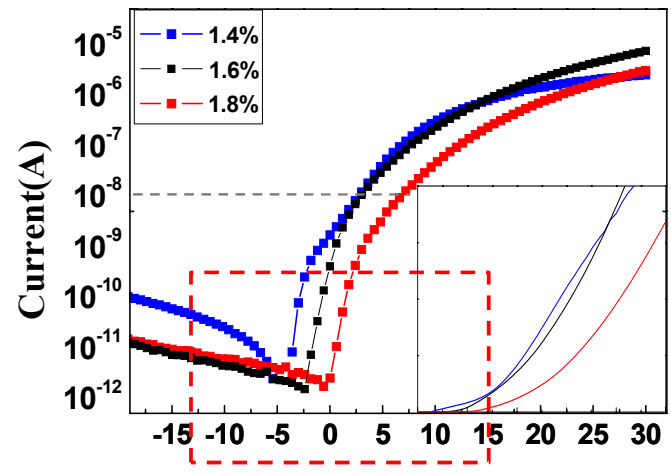

Fig. 3. Transfer curve of a-IGZO TFTs as a function of $\mathrm{O}_{2}$ partial pressure when the power density was 5.09 $\mathrm{W} / \mathrm{cm}^{2}$.

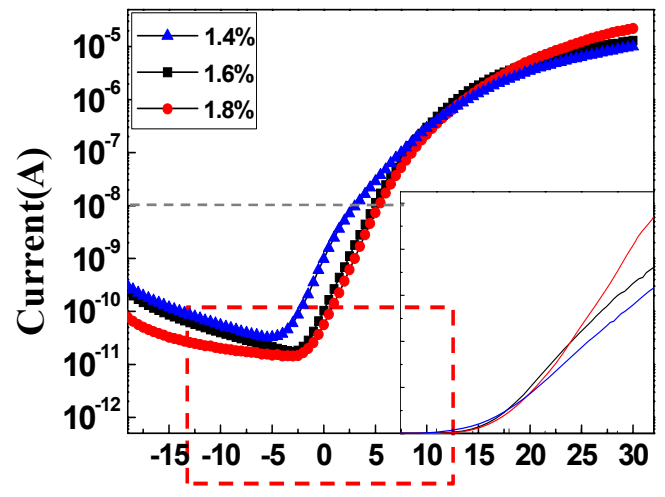

Fig. 4. Transfer curve of a-IGZO TFTs as a function of $\mathrm{O}_{2}$ partial pressure when the power density was 2.55 $\mathrm{W} / \mathrm{cm}^{2}$

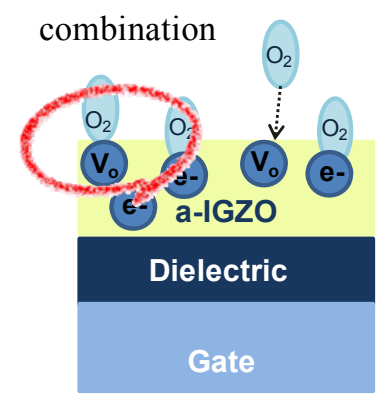

Fig. 5. Possible model of the electrical field-induced $V_{\text {th }}$ shift depending on $\mathrm{O}_{2}$ ratio.

is the measured drain current at $\mathrm{V}_{\mathrm{D}}=10 \mathrm{~V}$ and $\mathrm{V}_{\mathrm{G}}=10 \mathrm{~V}$, $\mathrm{L}$ is the gate length, $\mathrm{W}$ is the channel width, $\mathrm{d}$ is the gate insulator thickness, $\mathrm{q}$ is the electron charge, and $\mu_{F E}$ is the field effect mobility. The resulting carrier concentrations decreased with increasing $O_{2}$ ratio at $10^{16} \mathrm{~cm}^{-3}$ [21]. In fact, several studies have reported on controlling the electrical properties of the a-IGZO film using a combination of $\mathrm{O}_{2}$ and $O$ vacancies [18, 19]. This behavior shows similar attribution as that of a $p$-type dopant. This is because the oxygen vacancies compensated by $\mathrm{O}_{2}$ makes the channel layer less conductive in supplying free electron carriers to the conduction band. In other words, higher $\mathrm{O}_{2}$ ratio induces lower density of oxygen vacancies that usually act as shallow donors. The remaining donor electrons after compensating for the acceptors contribute to channel mobile carriers in the a-IGZO channel. Thus, the density of the remaining electrons of the deposited a-IGZO film should be lower in a high $\mathrm{O}_{2}$ ratio than in a low $\mathrm{O}_{2}$ ratio $[22,23]$. Moreover, the combination of the $\mathrm{O}_{2}$ and $\mathrm{O}$ vacancies influences the carrier concentration in a positive way. The carrier concentration of a-IGZO TFTs cannot be estimated in any measurable value range. Therefore, the positive $V_{t h}$ shift of the a-IGZO deposited at high $\mathrm{O}_{2}$ ratio ambience is understandable. The IZO-based TFTs with higher oxygen ratio shows the decrease of oxygen deficiencies, which can be clearly explained by observing the binding energy change of the O1s peak [24].

The a-IGZO TFTs with higher $\mathrm{O}_{2}$ ratio showed enhanced subthreshold-swing (S.S) value, indicating that oxygen suppressed the formation of trap center. This is because the $S . S$ value can explain the total trap density in the a-IGZO channel layer, and at the interface between the channel layer and gate insulator [24].

\section{Conclusion}

Oxide TFTs comprise a promising technology as an active device for large areas in terms of scalability, uniformity, and device performance. The effect of oxygen partial pressure on the transfer characteristics of a-IGZO TFTs has been investigated. It has been found that the oxide semiconductor properties are highly dependent on their oxygen content, because oxygen vacancies provide the needed free carriers for electrical conduction. The $\mathrm{O}_{2}$ partial pressure (or ratio) has been clearly observed to manipulate the $V_{t h}$ value of TFTs. The $V_{t h}$ value shifted in the positive direction as $\mathrm{O}_{2}$ ratio increased due to charge transfer on the oxide semiconductor. The a-IGZO films are properly made using adequate $\mathrm{O}_{2}$ partial pressure during the deposition process. The present work indicates that adjusting the a-IGZO channel layer depending on $\mathrm{O}_{2}$ partial pressure is feasible. The device performance is clearly affected by oxygen ambience due to the deposited film, which is closely related with the modulation generation achieved by reducing localized trapping carriers and defect centers at the interface or in the channel layer. In addition, the adjustable a-IGZO channel layer approach provides a significant potential to enhance the active matrix device performances.

\section{Acknowledgment}

This work was supported by the IT R\&D program of MKE/IITA [KI002182, TFT backplane technology for next generation display]. 


\section{References}

[1] E. G. Chong, K. C. Jo, S. Y. Lee, Appl. Phys. Lett. 96, (2010), 037015.

[2] P. Barquinha, A. Pimentel, A. Marques, L. Pereira, R. Martins, and E. Fortunato, J. Non-Cryst. Solids, 352, (2006), 1749.

[3] R. Martins, et al, J. Appl. Phys. 96, (2004), 1398.

[4] S. H. Jeong, B. N Park, D. G Yoo, J. H Boo, and D. G Jung, J. Korean Phys. Soc. 50, (2007), 3.

[5] K. W. Kim, P. C. Debnath, D. H. Park, S. S. Kim, and S. Y. Lee, Appl. Phys. Lett. 96, (2010), 083103.

[6] J. W. Kim, H. S. Kang, and S.Y. Lee, KIEE J. Electr. Eng. Technol. 1, (2006), 1, 98-100.

[7] B. S. Kim, D. E. Kim, G. C. Choi, J. W. Park, B. J. Lee and Y. S. Kwon, KIEE J. Electr. Eng. Technol. 4, (2009), 3, 418-422.

[8] E. Fortunato, A. Pimentel, A. Goncalves, A. Marques, R. Martins, Thin Solid Films. 502, (2006), 104.

[9] Y. K. Moon, S. Lee, J. W. Park, D. H. Kim, J. H. Lee and C. O Jeong, J. Korean Phys. Soc. 54, (2009), 1.

[10] C. H. Jung, D. J. Kim, Y. K. Kang, and D. H. Yoon, Jpn. J. Appl. Phys. 48, (2009).

[11] K. Nomura, H. Ohta, A. Takagi, T. Kamiya, M. Hirano, and H. Hosono, Nature (London). 432, (2004), 488.

[12] P. Barquinha, L. Pereira, G. Gonçalves, R. Martins, and E. Fortunato, J. Electrochemical Society. 156, (2009), H161.

[13] J. H. Jeong, H. W. Yang, J. S. Park, J. K. Jeong, Y. G Mo, H. D. Kim, J. Song, and C. S. Hwang, Electrochemical and Solid State Lett. 11, (2008), H157.

[14] H. Hosono, K. Nomura, Y. Ogo, T. Uruga, T. Kamiya, J. Non-Cryst. Solids. 354, (2008), 2796.

[15] J. S. Park, J. K. Jeong, Y. G. Mo, H. D. Kim, and C. J. Kim, Appl. Phys. Lett. 93, (2008), 033513.

[16] P. T. Liu, Y. T. Chou, and L. F. Teng, Appl. Phys. Lett. 95, 233504, 2009.

[17] C. Kilic, and A. Zunger, Appl. Phys. Lett., 81, 73, 2002.

[18] C. G. Van de Walle, Phys. Rev. Lett., 85, 1012, 2000.

[19] C. J. Park, Y. W. Kim, Y. J. Cho, S. M. Bobade and D. K. Choi, J. Korean Phys Soc. 55, (2009), 5.

[20] Y. Orikasa, M. Hayashi, and S. Muranaka, J. Appl. Phys. 103, (2008), 113703.

[21] Ibrahim Abdel-Motaleb, Neeraj Shetty, Kevin Leedy, and Rebecca Cortez, J. Appl. Phys. 109 (2011) 014503.

[22] B. Theys, V. Sallet, F. Jomard, A. Lusson, J.-F. Rommelue're, and Z. Teukam, J. Appl. Phys. 91, (2002), 3922.
[23] N. Ohashi, T. Ishigaki, N. Okada, T. Sekiguchi, I. Sakaguchi, and H. Haneda, Appl. Phys. Lett. 80, (2002), 2869.

[24] E. Chong, Y. S. Chun, and S. Y. Lee, Electrochem. Solid-State Lett., 14, (2011) H96.

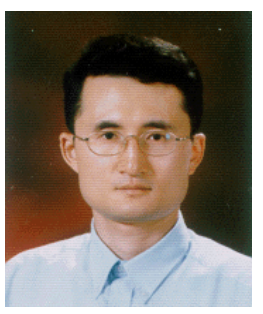

Sang Yeol Lee received his B.S. degree in Electrical Engineering from Yonsei University, and his M.S. and $\mathrm{Ph} . \mathrm{D}$. in Electrical Engineering from the State University of New York at Buffalo. $\mathrm{He}$ is a Professor of Nanoelectronics at the University of Science and Technology, Korea. His research interests include semiconductor processing, optoelectronic device, display device, memory device, and nano electronics. He is currently the Principal Research Scientist at the Electronic Materials Center, Korea Institute of Science and Technology (KIST).

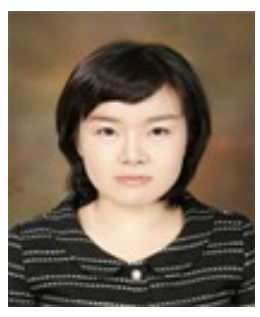

Eugene Chong received his M.S. degree in Material Engineering from the Inha University. Her research interests include $\mathrm{Zn}$-based oxide thin film transistor and their analysis. She is currently a Ph.D. candidate in Nanoelectronics at the University of Science and Technology.

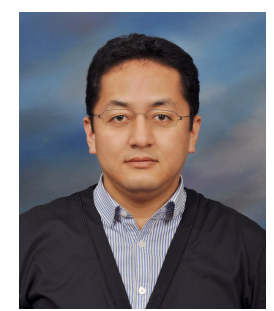

Yoon Soo Chun received his B.S. and M.S. degrees in Electrical Engineering from the Arizona State University at Tempe, and his Ph.D. degree in Materials Science and Engineering from the University of Washington. His research interests include oxide semiconductor, memory devices, and magnetic domains. He is currently in a post-doctoral position at the Electronic Materials Center in KIST.

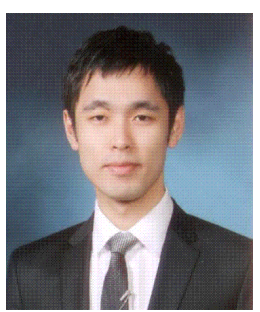

Seung Han Kim received his B.S. degree in Electrical and Electronics Engineering from Chung-Ang University. His research interests include semiconductor devices, circuit design, and plasma processing. 